\title{
Validation of the superconducting 3.9 GHz cavity package for the European X-ray Free Electron Laser
}

\author{
C. G. Maiano, ' J. Branlard, M. Hüning, K. Jensch, D. Kostin, A. Matheisen, W.-D. Möller, \\ A. Sulimov, and E. Vogel \\ Deutsches Elektronen-Synchrotron DESY, Notkestraße 85, 22607 Hamburg, Germany \\ A. Bosotti, J. F. Chen, M. Moretti, ${ }^{\dagger}$ R. Paparella, P. Pierini, ${ }^{*}$ and D. Sertore \\ INFN Sezione di Milano-Laboratorio LASA, Via Fratelli Cervi 201, 20090 Segrate (Mi), Italy
}

(Received 15 February 2017; published 24 April 2017)

\begin{abstract}
A full test of the cavity package concept under realistic operating condition was a necessary step before the assembly of the European XFEL (EXFEL) 3.9 GHz superconducting system and its installation in the accelerator. One cavity, equipped with magnetic shielding, power coupler and frequency tuner has been tested in a specially designed single cavity cryostat in one of the test benches of the DESY Accelerator Module Test Facility (AMTF). The cavity was operated at high pulsed power up to an accelerating field of $24 \mathrm{MV} / \mathrm{m}$, above the quench accelerating field of $21 \mathrm{MV} / \mathrm{m}$ achieved during the continuous wave $(\mathrm{CW})$ vertical qualification test and with a large margin with respect to the EXFEL maximum operating specification of $15 \mathrm{MV} / \mathrm{m}$ for the $3.9 \mathrm{GHz}$ system. All subsystems under test-coupler, tuner, waveguide tuners, low level radio-frequency (LLRF) system—were qualified to their design performances.
\end{abstract}

DOI: 10.1103/PhysRevAccelBeams.20.042005

\section{THE NEED FOR CAVITY PACKAGE VALIDATION}

The correct operation of a superconducting cavity in an accelerator under nominal conditions requires a number of active and passive subcomponents. Among these, a cold tuning system (CTS) is needed to finely adjust the cavity frequency to the master frequency reference of the facility; a fundamental power coupler (FPC) is used to deliver the necessary fundamental mode power for the cavity excitation and beam acceleration; a magnetic shield (MS) provides the necessary attenuation of the earth field to the superconducting material and the higher order modes (HOM) couplers transfer the power of the beam-excited wake fields out of the cold cavity (while properly notching the main accelerating mode frequency component); and a pick up antenna (PU) is used to probe the cavity field. In addition, the cryomodule mechanical environment around the cavity needs to allow for the relative differential thermal contraction of different materials without introducing uncontrolled forces on the cavities that could affect their natural resonant frequencies.

\footnotetext{
*Corresponding author. cecilia.giovanna.maiano@desy.de

Present address: CERN, Geneva, Switzerland.

On leave at DESY Hamburg, Germany.
}

Published by the American Physical Society under the terms of the Creative Commons Attribution 4.0 International license. Further distribution of this work must maintain attribution to the author(s) and the published article's title, journal citation, and DOI.
Frequently, in order to validate the complete design of the cavity package in its operating environment a complete test of a cavity dressed with all its ancillary components is performed in a short horizontal cryostat, which preferably provides a mechanical environment similar to that of the cryomodules in the accelerator [1,2]. Such an activity took place at the DESY AMTF facility, in joint collaboration between DESY and INFN, for the characterization of the EXFEL 3.9 GHz cavity package.

\section{THE EUROPEAN XFEL 3.9 GHZ SYSTEM}

The European XFEL injector hosts a cryomodule composed of 8 superconducting radio-frequency (SCRF) cavities resonating at $3.9 \mathrm{GHz}$, to manipulate the longitudinal beam phase space before the three bunch compressor stages occurring downstream along the linac [3-5]. The module design has been derived from the third-harmonic section at FLASH, developed by FNAL [6], with some major modifications to the cavity package design, in particular in order to provide alternate coupler orientation with respect to the beamline, for dipole coupler kick cancellation [7]. The cavities have been procured by INFN and vertically tested at the LASA laboratory [8] and the module has been jointly prepared by INFN and DESY for tunnel installation, as described in several contributions to various particle accelerator conference proceedings [9-12].

Figure 1 shows a 3D model of the $3.9 \mathrm{GHz}$ dressed cavities (illustrating both coupler orientation variants as foreseen in the accelerator module), equipped with the cold tuners, the cold fundamental power coupler part, the low 


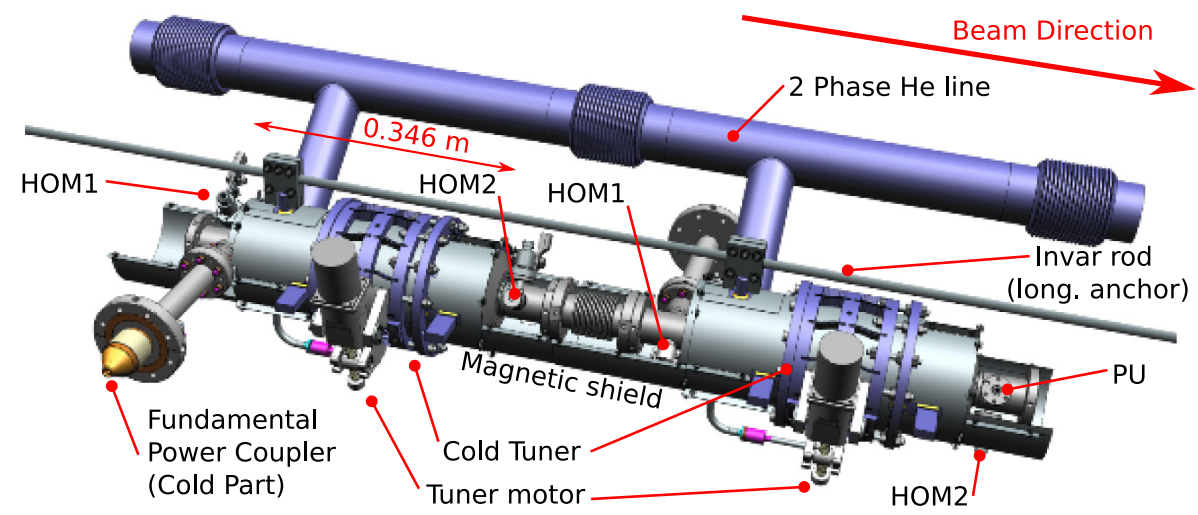

FIG. 1. The two EXFEL 3.9 GHz cavity packages in the cavity string, with opposing coupler orientations.

coupling radio frequency antennas for the field pick-up probe and HOMs, and the magnetic shield (the top half of the parts connecting the cavities and the support structure of the module have been blanked for clarity).

\section{THE AMTF CRYOADAPTERS}

All EXFEL $1.3 \mathrm{GHz}$ main linac modules were qualified in the DESY AMTF after their assembly [13]. The qualification test foresees the individual cavity performance characterization and the procedure includes the verification of the correct functionality of all cavity ancillaries [14]. After the successful outcome of the AMTF test each module was then equipped with its individually tailored rf distribution system and prepared for the installation in the tunnel. The third harmonic system was technically commissioned and characterized by means of rf measurements performed directly in the injector building after installation. This was decided to limit the infrastructure work needed for adapting the AMTF for the testing of $3.9 \mathrm{GHz}$ modules, to avoid slowing down the test schedule of the $1011.3 \mathrm{GHz}$ modules needed for the EXFEL linac, and considering the moderate performances needed by the $3.9 \mathrm{GHz}$ cavities [5]. Due to this decision, the qualification of the cavity package in a horizontal test represented an important verification needed well before the start of the module assembly activities.

Even if no horizontal tests of single cavities were envisaged during the EXFEL construction, two short $(\approx 2.2$ meter) cryostats fitting the cryogenic connections of the AMTF test caves were delivered by the Budker Institute of Nuclear Physics (BINP) as part of the Russian in-kind contribution to EXFEL [15]. These adapters will allow future testing and R\&D activities on single $1.3 \mathrm{GHz}$ cavities in a mechanical environment close to their operating conditions.

In order to establish testing capabilities for the $3.9 \mathrm{GHz}$ cavities at the AMTF in DESY, one of the cryoadapters has been extensively modified in collaboration with BINP, adding a second fundamental power coupler port on the opposite side of the existing one (to allow the installation of both $3.9 \mathrm{GHz}$ cavity package variants) and providing a new internal cavity sliding suspension support, based on the geometry of the $3.9 \mathrm{GHz}$ module developed at INFN-LASA. Figure 2 shows the cavity supported through the sliding roller bearings to the stainless steel tray inside the adapter. Additionally, one of the EXFEL accelerator test benches (XATB1) in the AMTF at DESY has been equipped with the rf infrastructure needed to test cavities at $3.9 \mathrm{GHz}$ (a $80 \mathrm{~kW}$ klystron, a modulator to provide the nominal EXFEL pulse, a waveguide system, LLRF/ technical interlock crates and motor driver racks for the actuation of the cavity frequency tuner and coupler stub

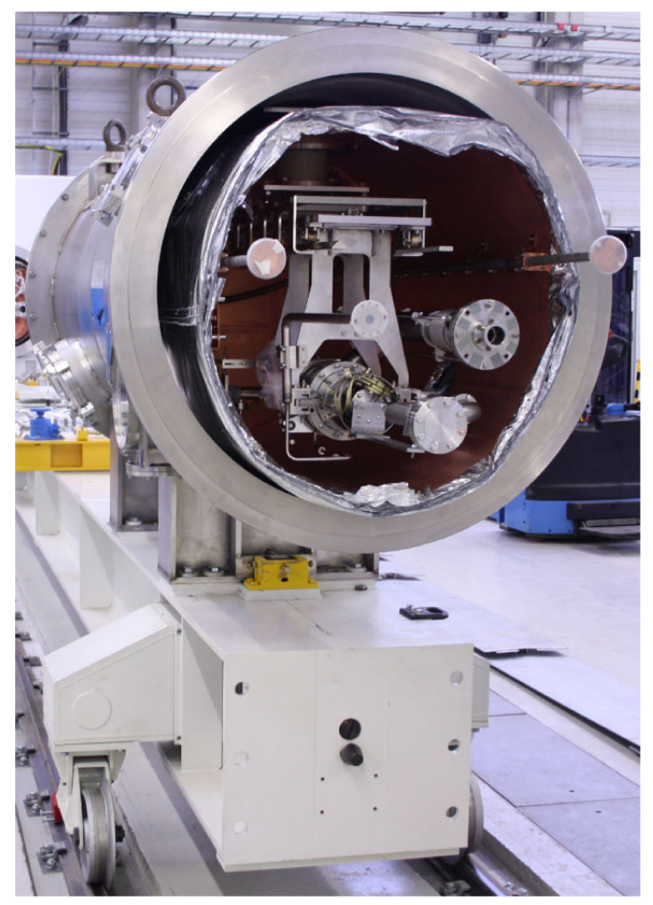

FIG. 2. The cavity package installed in the cryoadapter before connection to the XATB1 cryogenic feedbox. The cavity is supported by a sliding mechanism to the stainless steel suspension support, which reproduces the mechanical installation in the accelerating module cryostat. 
tuner). The standard diagnostic crates of the XATB1 used for the $1.3 \mathrm{GHz}$ module testing allow the monitoring or all the module signals (cavity and coupler temperatures and the rf signal sampling from the cavity rf ports).

The test activities were performed in collaboration between INFN-LASA and DESY, which jointly developed the $3.9 \mathrm{GHz}$ system as an in-kind contribution to the EXFEL Project [16].

\section{THE VALIDATION TEST}

\section{A. Motivations}

While the electromagnetic design of the EXFEL 3.9 GHz cavity has not been altered with respect to the cavities used in the FLASH ACC39 module [6], several important modifications have been implemented in the cavity package design, including important mechanical details of the cavity and its titanium helium vessel, and a complete revision of all ancillary components. The differences between the EXFEL design and the original cavity design by FNAL [17] are extensively described in Ref. [8].

To avoid emittance dilution in the injector, beam dynamics studies indicated that a scheme for coupler dipole kick compensation was required, resulting in a string with an alternate orientation of the couplers with respect to the beamline, as shown in Fig. 1. Two versions of the dressed cavity assembly, differing only in the relative rotation of 180-degree of the cavity around its axis during integration in its vessel, allow for such an arrangement.

Concerning the ancillaries, a scaled version of the ILC blade tuner CTS [18] was developed for the EXFEL 3.9 GHz cavities [19] to profit from its smaller size with respect to the ACC39 CTS and to allow installation with the lateral 2-phase circuit position of the EXFEL modules. The roller bearing pads [20] needed for the string support from the helium gas return pipe (that acts as the structural backbone of the accelerating module) have been moved to a different position in the tank, no longer attached to the tuner disks, but now directly welded to it, which required the development of a new compact roller bearing assembly. The two cavity variants required the development of a different magnetic shield design, fitting both cavity orientations.

Finally, the different EXFEL beam parameters required a setting for the coupler loaded quality factor $\left(Q_{L}\right)$ of nearly twice the FLASH value. This was achieved with a different length of the coupler antenna.

The several changes performed on the cavity package with respect to the ACC 39 design motivated the need of a validation test of a complete unit. The test was performed to demonstrate the ability to reach the nominal performances before proceeding to the EXFEL $3.9 \mathrm{GHz}$ system assembly.

\section{B. Selection of the test cavity}

One of the cavities produced for the installation into the EXFEL 3.9 GHz system, cavity 3HZ010, was selected and prepared for the horizontal test in the AMTF cryoadapter prior to its use in the module. The cavity was vertically tested at INFN-LASA in September 2014, during the test campaign of the series of the ten cavities produced for the 3.9 GHz string [10]. The vertical test (VT), performed in a bath cryostat at $2 \mathrm{~K}$, is meant to qualify the cavity in quasi$\mathrm{CW}$ conditions (with $25 \% \mathrm{RF}$ duty cycle at $0.5 \mathrm{~Hz}$ to limit cryogenic losses and HOM heating) up to the nominal accelerating gradient and quality factor set by the project: $E_{\text {acc }}=15 \mathrm{MV} / \mathrm{m}$ and $Q_{0}=10^{9}$.

All the cavities were tested before the $\mathrm{He}$ vessel integration [8,9], equipped with a fixed high- $Q$ antenna (i.e., close to the critical coupling conditions for the cavity), a field pick up and two HOM antennas (one at both cavity ends), and after the successful tuning of the HOM coupler notch filters for the rejection of the fundamental cavity mode. The maximum accelerating field $E_{\text {acc }}$ in the test was reached close to a quench occurring at approximately $21 \mathrm{MV} / \mathrm{m}$, with the cavity showing a $Q_{0}$ of $\approx 2 \times 10^{9}$ with no decrease from low field levels up to the quench limit. No field emission was detected. The cavity thus achieved its full qualification and proceeded to the $\mathrm{He}$ vessel integration and to its preparation for the installation in the module.

\section{He vessel integration and assembly of the ancillaries}

The integration tool used for the welding of the titanium He vessel on the cavity ensures that any material shrinkage occurring during welding does not have a chance to mechanically load the constrained Niobium structure, avoiding the possibility to induce permanent deformations which would cause an uncontrolled detuning from its goal frequency. Additionally, the integration was performed under frequency control with a vector network analyzer (VNA) during all critical stages of the welding process.

After the vessel integration, the installation of the pick up and HOM antennas and the high pressure rinse in the LASA clean room, the cavity was sent from LASA to DESY in vacuum conditions (pressure $<10^{-8}$ mbar), ready to be dressed for the vertical test. At DESY the standard mechanical and rf incoming tests were performed upon acceptance and the cavity entered in the clean room after degreasing and rinsing of the external surfaces, for the venting and installation of the FPC and the pump manifold to connect to the active pumping system of the test stand. No further treatment or cleaning of the internal rf surfaces was performed at DESY.

The complete installation of the cavity ancillaries (i.e., fundamental power coupler, the cold magnetic shield, and the cold frequency tuner) was then performed at the DESY cavity preparation infrastructure. Any undesired cavity elongation or contraction that could be induced by mechanical assemblies, as the tuner or magnetic shield installation, was performed under the control of the stability of its resonant frequency, within a few tens of $\mathrm{kHz}$. 


\section{Warm rf preparation procedures}

The $3.9 \mathrm{GHz}$ cavity tuner (blade type) is designed to elastically extend the cavity, inducing a positive frequency shift of about $180 \mathrm{kHz}$ for each turn of the motor spindle, for a potential maximal tuning range of about $1.5 \mathrm{MHz}$ at $0.7 \mathrm{~mm}$ elongation [19]. Cavities were tuned during the last fabrication stages to reach at cold a frequency approximately $400-500 \mathrm{kHz}$ below the operation frequency of $3.9 \mathrm{GHz}$, in order to avoid tuner operation around its mechanical neutral point, thus avoiding a potentially significant residual hysteresis. After tuner installation, the cavity was transferred on the cryoadapter internal suspension support. The support was then inserted in the vacuum vessel and a check of the tuner operation was performed a few times, on a short range (single turn of the motor spindle), to verify the correct assembly operation and the proper frequency response of the cavity. A larger excursion of the tuner action at warm may induce permanent plastic deformation on the resonator, resulting in an uncontrolled detuning from the goal frequency. The cavity installed in its suspended position in the cryoadapter is shown in Fig. 2.

The results of the tuner operation at warm, after inserting the cavity support in the cryostat adapter, are shown in Table I.

Before installation in the cryostat adapter the transmissions across all opposed cavity ports-i.e., power coupler pick-up (FPC-PU), power coupler HOM2 (FPC-HOM2), and pick-up HOM1 (PU-HOM1), where HOM1 is the HOM antenna at the FPC side and HOM2 at the PU side-were checked. After any mechanical operation around the cavity (e.g., the assembly of the tuner and magnetic shield) a detuning of the HOM coupler notch filters is possible, due to their high sensitivity with respect to mechanical deformations. Thus the whole fundamental passband spectrum was measured to verify the proper HOM transfer functions and the correct position of the notch filters for the rejection of the fundamental mode.

The main effect experienced in several cavities during the module preparation is the detuning of HOM1, (the one which directly faces the FPC port), due to the installation of the FPC antenna, which slightly changes the field pattern in the end region of the cavity with respect to the high- $Q$ antenna case used for the vertical tests. Figure 3 shows the evolution of the HOM relative transmissions, starting from the $3 \mathrm{HZO10}$ cavity reception at DESY with the high- $Q$

TABLE I. Tuner operation at warm. Each test cycle consists of a full motor spindle turn followed by the return to the initial position.

\begin{tabular}{lcc}
\hline \hline Motor action & Frequency shift/turn & Hysteresis \\
\hline First cycle & $172 \mathrm{kHz}$ & $<1 \mathrm{kHz}$ \\
Second cycle & $177 \mathrm{kHz}$ & $<1 \mathrm{kHz}$ \\
\hline \hline
\end{tabular}
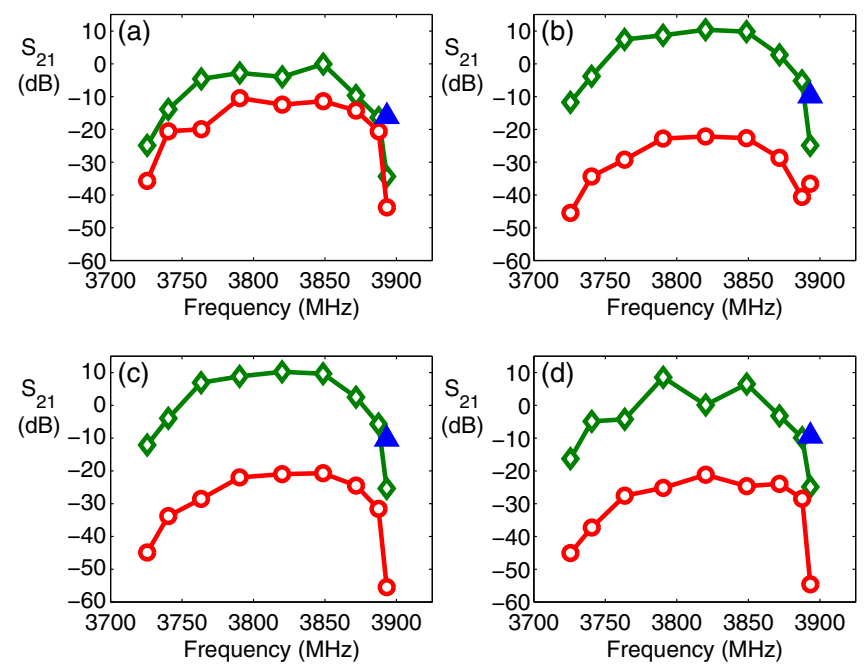

FIG. 3. Evolution of the relative HOM transmission spectra of the fundamental passband modes, from cavity reception (inset a), FPC installation (inset $b$, showing the detuning of the HOM1 notch filter), HOM retuning (inset c) and after installation of all ancillaries (inset d). In each plot, diamonds show the FPC-HOM2 passband, circles the PU-HOM1 passband and the triangle indicates the fundamental mode FPC-PU transmission.

fixed antenna (inset a) and following its preparation stages for the horizontal test (insets $b$ to d). The detuning of the HOM1 coupler notch filter position after the FPC installation (inset $b$ ) required to perform the retuning operation shown in inset c, whereas the analysis of the relative transmissions measured before and after the installation of the CTS and MS show that these operations did not induce any detuning of the notch filter positions (as shown in inset d).

Although no operational experience existed with these cavities at the time of the qualification test, the specifications for a satisfactory notch filter tuning were set to the following requirements: (I) a transmission from FPCHOM2 at least $10 \mathrm{~dB}$ lower than the FPC-PU on the fundamental mode and (II) a PU-HOM1/FPC-HOM2 transmission on the fundamental mode less than $20 \mathrm{~dB}$ than its maximum on the fundamental bandwidth and $10 \mathrm{~dB}$ less than the neighboring $(8 \pi / 9)$ mode. These requirements are satisfied in the last set of curves shown in Fig. 3 after the installation of all cavity ancillaries, right after the connection of the cryostat adapter to the cryogenic cap. During VT no substantial HOM power leakage of the fundamental mode was observed after the notch filter tuning. These conservative criteria will be updated when the full experience of the EXFEL $3.9 \mathrm{GHz}$ system becomes available.

\section{E. Installation in the XATB1 test bench}

The cavity 3 HZO10 is supported in the cryostat adapter through a sliding mechanism using spring-loaded linear roller bearings similar to those used in the cryomodule for 


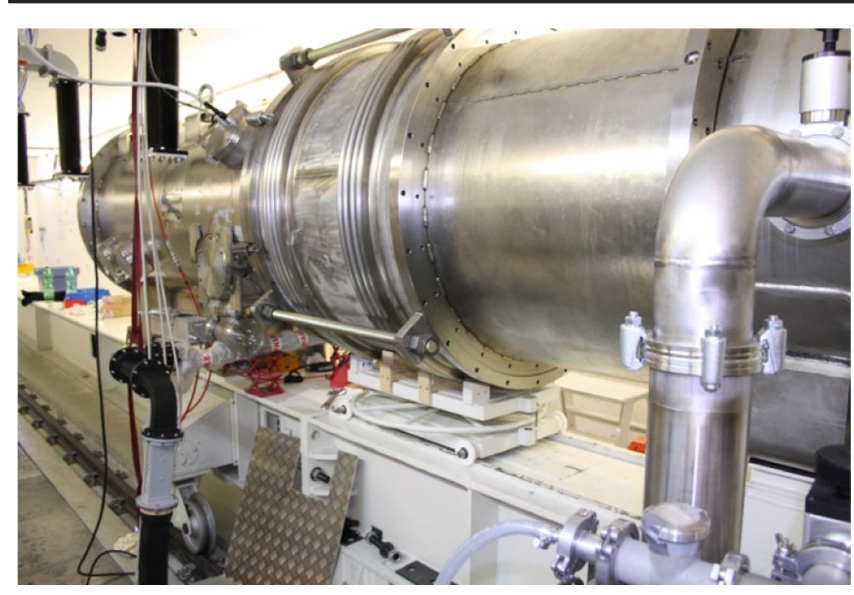

FIG. 4. The cryostat adapter during its connection to the XATB1 cryogenic feedbox.

operation [20], allowing the differential thermal contraction of the $\mathrm{Nb} / \mathrm{Ti}$ cavity assembly with respect to the stainless steel support, while maintaining the coupler longitudinal position fixed at the warm module port. The warm coupler components were assembled using a local clean room after the installation of the cavity in the adapter.

The cryoadapter has a single thermal shield connected to the 40-70 K circuit and the cavity suspension support slides on a fixed rail suspended from fiberglass posts and thermalized by the 5-8 K circuit of the XATB (See Fig. 2). All rf and temperature sensor connections are routed to patch panels in the adapter to reduce the setup test time.

After the assembly of the cavity in the adapter the vacuum vessel was moved onto the cryomodule support rail of XATB1 and rolled into the test cave to connect the cryogenic circuits, the cavity beam vacuum line and the 3.9 GHz waveguides from the klystron to the warm coupler waveguide box. The insulation vacuum was then closed using the large feed cap sliding bellow and all circuits were leak checked. Figure 4 shows the cryoadapter connected to the feed box.

\section{CAVITY PACKAGE TESTING}

\section{A. Cooldown and warmup cycle}

Cooldown started on March 19, 2015, with the standard fast cooling procedures used for the main linac module tests in AMTF [13], where process pipes are cooled down to the nominal temperatures in approximately 14 hours. Figure 5 shows the inlet and outlet temperatures of the thermal shields and the temperature of the cold box in the feed cap which provides the 2 phase He to the $2 \mathrm{~K}$ cavity line. After the full thermalization of the cold mass the pump-down to subatmospheric pressure to reach the $2 \mathrm{~K}$ operation started on March 23, 2015, followed by the cold tuning and low power measurements for calibration. High power operation and LLRF tests started on March 25, 2015. Warmup started on March 27, 2015.

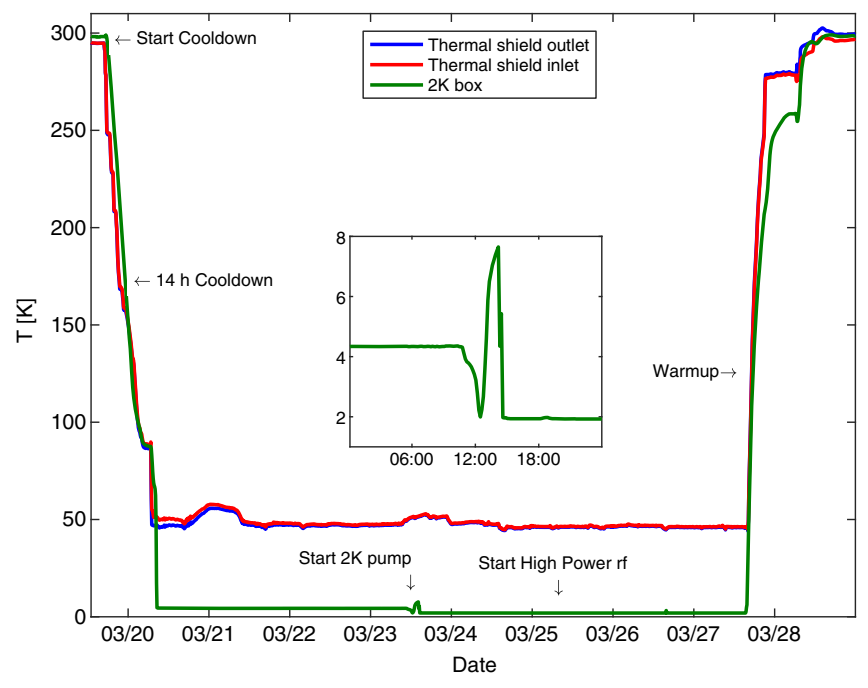

FIG. 5. Cooldown/warmup cycle of the cryoadapter for the $3.9 \mathrm{GHz}$ horizontal tests. The cryogenic circuits are cooled and warmed with a standard procedure requiring approximately $14 \mathrm{~h}$. More than 48 hours were needed for complete thermalization of the cold mass before the $2 \mathrm{~K}$ pump down.

\section{B. Cold rf preparation procedures}

The warm-to-cold frequency shift experienced during all vertical tests of the cavities before integration was $6.1 \pm 0.1 \mathrm{MHz}$ [9]. The frequency of 3HZ010 after cooldown increased by $6.17 \mathrm{MHz}$, in full agreement with the VT experience and indicating that tank integration and the cavity support scheme in the module lead to no undesirable or uncontrolled stresses on the rf structure due to differential material shrinkage. The cavity tuning and preparation scheme foresees a target frequency approximately $500 \mathrm{kHz}$ lower than the operation frequency, in order to always operate the blade tuner far from its mechanical neutral (rest) point, as discussed previously.

After reaching a stable $2 \mathrm{~K}$ cavity condition, the cavity was tuned to $3.9 \mathrm{GHz}$ by the CTS action under the control of a VNA, to monitor the cold tuning action and to verify the tuner performance. Each motor screw turn requires

TABLE II. $3 \mathrm{HZO10}$ frequency history and tuner parameters.

\begin{tabular}{lc}
\hline \hline Cavity status & Frequency \\
\hline Warm frequency before cooldown & $3893.279 \mathrm{MHz}$ \\
Cold frequency after cooldown & $3899.449 \mathrm{MHz}$ \\
Cold-warm frequency Shift & $+6.17 \mathrm{MHz}$ \\
\hline \hline Tuner parameter & Value \\
\hline Motor turns to $3.9 \mathrm{GHz}$ & $\approx 3.5$ \\
Motor steps to tune position & $\approx 246200$ \\
Frequency offset/turn & $171 \mathrm{kHz}(1 \mathrm{st}$ turn $)$ \\
& $166 \mathrm{kHz}(2 \mathrm{nd} \mathrm{turn})$ \\
Frequency offset/motor step & $160 \mathrm{kHz}(3 \mathrm{rd} \mathrm{turn})$ \\
\hline \hline
\end{tabular}



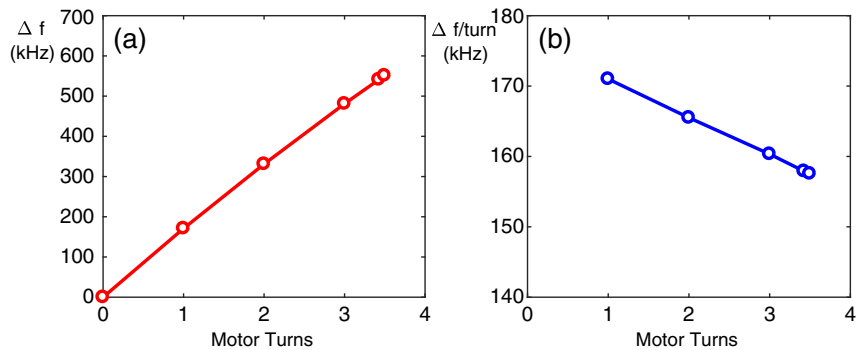

FIG. 6. Cavity frequency offset (left) and frequency offset per motor turn (right) during the tuning process.

70400 steps of the stepper motor and the cavity was brought to the frequency target with 246200 steps, corresponding to roughly 3.5 screw turns and providing the necessary $551 \mathrm{kHz}$ of tuning action. The maximal tuning stroke for the tuner design is obtained by 15 screw turns, corresponding to a frequency tuning action in excess of $1.5 \mathrm{MHz}$ [19], which is still expected to be below the onset of cavity plasticity in cold condition.

Table II summarizes the cold-warm cavity frequencies and the tuner characterization results.

Figure 6 shows the cavity frequency shift from the unloaded rest position of the CTS and the frequency offset/ turn evolution during the tuning process.

After bringing the tuner back to its original position the cavity restored its original frequency: no hysteresis effects below the $10-20 \mathrm{kHz}$ range could be measured with a VNA given the relatively large noise environment in the low transmission measurement performed through the rf sampling channels of the AMTF facility.

\section{LOW POWER MEASUREMENTS AND CALIBRATION}

The accelerating gradient is one of the key parameters to qualify the cavity performance, thus the cavity $Q_{L}$ and the constant $k_{t}$ relating accelerating gradient to the probe power were determined at moderate forward power from the fundamental power coupler $\left(P_{f} \approx 1 \mathrm{~kW}\right)$, by measuring the decay constant $\tau$ of the transmitted probe power $P_{t}$ and estimating the accelerating field $E_{\text {acc }}$ from the forward power $P_{f}$ to the cavity driven with a rectangular rf calibration pulse (see Fig. 7). In detail $E_{\text {acc }}$ is estimated by:

$$
E_{\mathrm{acc}}=\frac{1}{L_{\mathrm{eff}}} \sqrt{\frac{R}{Q} 4 Q_{L} P_{f}}\left(1-\exp \left(\frac{t}{2 \tau}\right)\right)
$$

where $L_{\text {eff }}$ is the cavity rf length, $R / Q$ is the cavity shunt impedance of $750 \Omega$ for $3.9 \mathrm{GHz}$ cavities, $Q_{L}=\pi f \tau$ and $f$ is the cavity frequency. Therefore the constant $k_{t}$ is computed by $k_{t}=E_{\text {acc }} / \sqrt{P_{t}}$ at this calibration point. Subsequently, the cavity accelerating field is determined from the measured transmitted probe power $P_{t}$ with the simple relation $\left.E_{\text {acc }}[\mathrm{V} / \mathrm{m}]=k_{t} \sqrt{P_{t}[\mathrm{~W}]}\right)$.

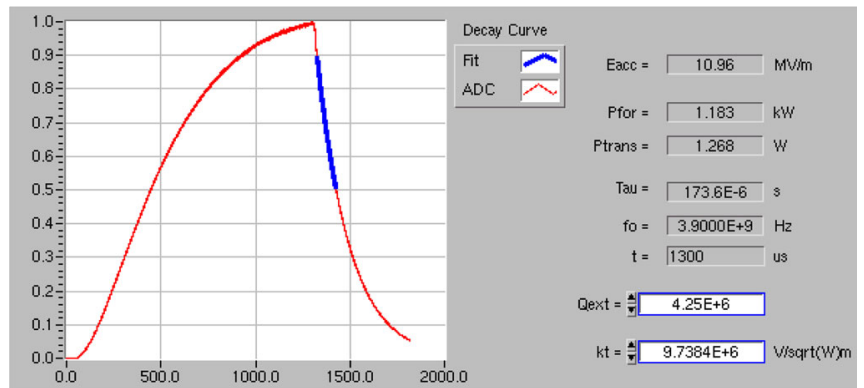

FIG. 7. Cavity transmitted power (shown in arbitrary units) using a rectangular low power rf pulse with $1.3 \mathrm{~ms}$ length. The bold (blue) region shows the portion of the pulse used for decay time and $Q_{L}$ evaluation.

The $3.9 \mathrm{GHz}$ coupler is of the fixed coupling type [21], and the nominal EXFEL beam parameters require a coupling with $Q_{L}=3.2 \times 10^{6}$, i.e., nearly twice the design value the FLASH third-harmonic system [22]. Thus each coupler antenna was trimmed to a different length with respect to the original design, based on the extrapolation of the FNAL antenna trimming experience. Three stub tuners on the waveguides allow for fine tuning of the coupling factor, at least a factor of 2 in both directions, and the adjustment of the relative cavity phases for correct beam operation under vector sum control in the accelerating modules. The measurement of the 3HZ010 cavity yielded a value of $Q_{L}=4.25 \times 10^{6}$, well within the adjustment range.

Table III summarizes the calibrations performed during the $3 \mathrm{HZ} 010$ test, for the external quality factor, $Q_{\text {ext }}$, of all antennas and for $k_{t}$, with comparison to the values determined during the VT at LASA. The comparison is within acceptable limits and, in particular, within the specifications (which were for the pick up a factor of ten above the nominal cavity quality factor, $Q_{0}^{\text {spec }}=10^{9}$, and for the HOM a corresponding factor of 100).

\section{A. High power measurements}

The cavity was then operated in open loop with $10 \mathrm{~Hz}$ pulses with a filling time of $500 \mu$ s and a flat-top time of $600 \mu \mathrm{s}$. The cavity was fine tuned in frequency under the control of the LLRF system (which is synchronized to the master reference system) by maximizing the transmitted power on the LLRF control panel, after which the input

TABLE III. Comparison of rf calibrations in the vertical and horizontal tests.

\begin{tabular}{lcc}
\hline \hline Item & VT & HT \\
\hline$Q_{\text {PU }}$ & $1.3 \times 10^{10}$ & $1.5 \times 10^{10}$ \\
$Q_{\mathrm{HOM} 1}$ & $8.8 \times 10^{13}$ & $2.2 \times 10^{13}$ \\
$Q_{\mathrm{HOM} 2}$ & $2.5 \times 10^{12}$ & $1.5 \times 10^{13}$ \\
$k_{t}$, in $\mathrm{V} /(\mathrm{m} \sqrt{\mathrm{W}})$ & $9.16 \times 10^{6}$ & $9.74 \times 10^{6}$ \\
\hline \hline
\end{tabular}




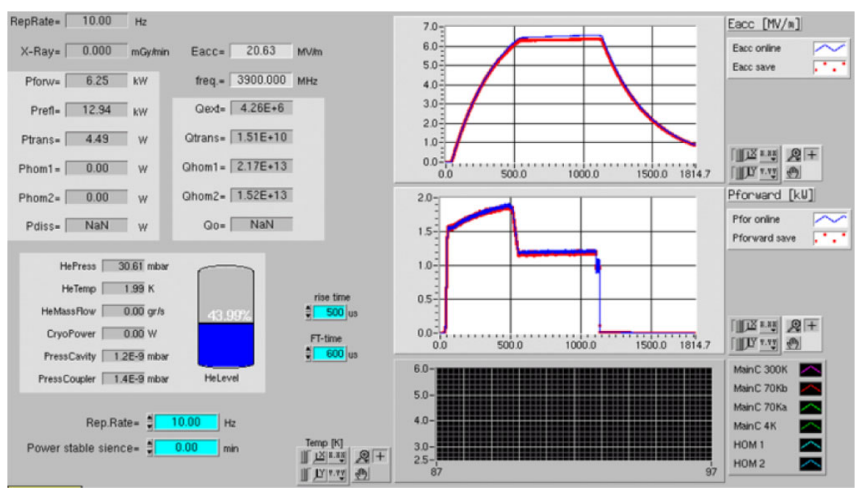

FIG. 8. Typical pulse measured during the long stable operation at $20 \mathrm{MV} / \mathrm{m}, 10 \mathrm{~Hz}$ operation with a $0.5 \mathrm{~ms}$ fill time and $0.6 \mathrm{~ms}$ flat top pulse in feed forward mode. $E_{\text {acc }}$ (top plot) and forward power (bottom plot) are displayed in arbitrary units. Power readings and computed $Q_{\text {ext }}$ values are shown in the left part of the control system panel.

power was raised to bring the cavity to its quench limit, before the cavity reached $24 \mathrm{MV} / \mathrm{m}$, above the quench level experienced in the vertical tests $(21 \mathrm{MV} / \mathrm{m})$ under quasi-CW conditions.

Stable operation was kept at $20 \mathrm{MV} / \mathrm{m}$ for nearly 48 hours in feed forward mode using a fill time of $500 \mu \mathrm{s}$ and a flat top of $600 \mu \mathrm{s}$. No appreciable sign of field emission was measured. Two gamma ray detectors for field emission detection are placed near the beam line on both ends of the module (by the end caps). No cavity heating was measured by the 8 CERNOX sensors positioned on the cavity vessel ( 2 on both cavity sides) and in three positions at each HOM coupler. A typical pulse measurement is shown in Figure 8.

\section{B. Comparison of vertical and horizontal test performances}

The stable $2 \mathrm{~K}$ operation of the small cryostat adapter in the AMTF required a $25 \mathrm{~W}$ offset for the cold box heater, as the cryogenic infrastructure has been designed to absorb the high heat load generated by a 8 cavity module of the EXFEL main linac. The average cavity dissipated power at $20 \mathrm{MV} / \mathrm{m}$ is only $0.35 \mathrm{~W}$ at $1 \%$ rf duty cycle at the $Q_{0}$ measured during the vertical test, therefore no precise measurements of dynamic heat losses are possible. Still a rough estimation of the cavity dynamic load could be performed in two different setups: the first keeping the He level control and evaluating the heater offset difference from rf-on and rf-off states; the second using a precise mass flow controller, switching off the heaters and closing the Joule-Thompson valve (i.e., running with decreasing $\mathrm{He}$ level, so-called boil-off test). These two methods, close to their measurement limits, indicated values of $0.2 \mathrm{~W}$ and $0.6 \mathrm{~W}$, respectively, compatible with the estimation of the cavity dissipation given above by means of the extrapolation of the VT results, and with the large uncertainty of the

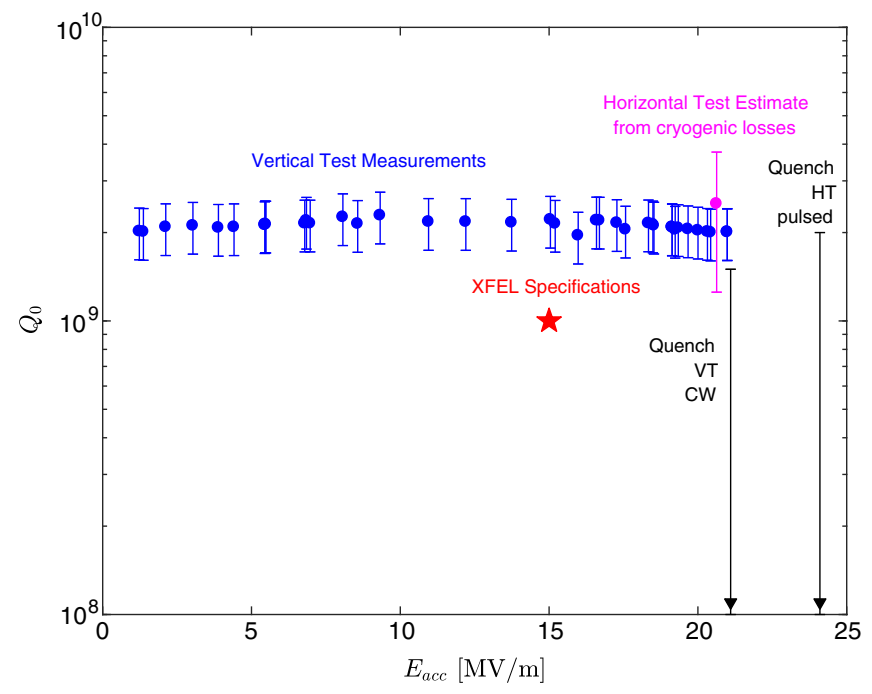

FIG. 9. Comparison between the vertical test measurements of the cavity at INFN-LASA and the qualification test in the horizontal cryostat at the AMTF.

setup. In both cases the measurement has an error close to $100 \%$ (the heater on and off method required a $0.2 \mathrm{~W}$ difference over the $25 \mathrm{~W}$ offset and in the boil-off method a difference of $0.03 \mathrm{~g} / \mathrm{s}$ was measured on top of a fluctuation of $0.02 \mathrm{~g} / \mathrm{s}$ in the rf-on and rf-off states).

Figure 9 shows the comparison of the quality factor $Q_{0}$ vs the accelerating voltage $E_{\text {acc }}$ obtained during the vertical characterization test at LASA with the analysis of the measurement obtained during the horizontal testing.

\section{C. $Q_{L}$ adjustment}

The 3-stub tuner at the waveguide was used to change the system $Q_{L}$ values, with more than a factor 2 variation being achieved, reaching a maximum $Q_{L} \approx 10^{7}$. However, the central step-motor connection was damaged during the installation phase and inoperative during the test. Hence the system could not be used to set to the nominal design value of $Q_{L}=3.2 \times 10^{6}$.

\section{CONCLUSIONS}

The cavity package for the $3.9 \mathrm{GHz}$ EXFEL system was fully qualified in a horizontal test in the AMTF facility at DESY. This activity was necessary before the start of the installation activities for the full system, due to the several modifications introduced in the cavity and ancillaries design with respect to the FLASH system. The qualification allowed the verification of the correct operation of all subsystems and the readiness of the cavity preparation and measurement procedures.

\section{ACKNOWLEDGMENTS}

We would like to thank our colleagues at DESY (groups MHFsl, MKS1, MKS3, MIN, MSK, and MVS) and at 
INFN-LASA for the support of the activities leading to the successful qualification of the EXFEL $3.9 \mathrm{GHz}$ cavity package. We acknowledge the support from Elvin Harms, Helen Edwards and FNAL colleagues for sharing the FLASH ACC39 design details and for the coupler preparation work, performed under a Work for Others agreement between DESY and FNAL. Colleagues at BINP provided the cryostat adapter design modifications and their implementation on the existing hardware to enable the tests described here.

[1] J. Kuzminski, First Cold Test of TESLA Superconducting RF Cavity in Horizontal Cryostat (CHECHIA), in Proceedings of International Conference on RF Superconductivity (SRF1995), Gif s. Yvette, France, 1995 (JACoW, Geneva, Switzerland, 1995), p. 455.

[2] T. Khabiboulline, H. Edwards, M. Foley, E. Harms, A. Hocker, D. Mitchell, A. Rowe, and N. Solyak, Vertical and Horizontal Test Results of $3.9 \mathrm{GHz}$ Accelerating Cavities at FNAL, in Proceedings of Eleventh European Particle Accelerator Conference (EPAC'08), Genova, Italy, 2008 (JACoW, Geneva, Switzerland, 2008), p. 874.

[3] R. Abela et al., XFEL: The European X-Ray Free-Electron Laser-Technical Design Report (DESY, Hamburg, 2006).

[4] W. Decking and T. Limberg, European XFEL Post-TDR Description, DESY Technical Report No. XFEL.EU TN2013-004, 2013.

[5] P. Pierini et al., Preparation of the $3.9 \mathrm{GHz}$ System for the European XFEL Injector Commissioning, in Proceedings of International Conference on RF Superconductivity (SRF2015), Whistler, BC, Canada, 2015 (JACoW, Geneva, Switzerland, 2015), p. 584.

[6] E. Harms, H. Edwards, M. Hüning, and E. Vogel, Commissioning and Early Operating Experience of the FLASH Third Harmonic RF System, in Proceedings of Linear Accelerator Conference (LINAC10), Tsukuba, Japan, 2010 (JACoW, Geneva, Switzerland, 2010), p. 422.

[7] P. Pierini, A. Bosotti, N. Panzeri, D. Sertore, H. Edwards, M. Foley, E. Harms, D. Mitchell, J. Iversen, W. Singer, and E. Vogel, Third Harmonic Superconducting Cavity Prototypes for the XFEL, in Proceedings of Linear Accelerator Conference (LINACO8), Victoria, BC, Canada, 2008 (JACoW, Geneva, Switzerland, 2008), p. 821.

[8] P. Pierini, M. Bertucci, A. Bosotti, J. F. Chen, C. G. Maiano, P. Michelato, L. Monaco, M. Moretti, C. Pagani, R. Paparella, D. Sertore, and E. Vogel, Fabrication and vertical test experience of the European x-ray Free Electron Laser $3.9 \mathrm{GHz}$ superconducting cavities (to be published).

[9] P. Pierini, M. Bertucci, A. Bosotti, J. Chen, A. Gresele, C. Maiano, P. Michelato, L. Monaco, M. Moretti, C. Pagani, R. Paparella, P. Pierini, M. Rizzi, D. Sertore, and E. Vogel, Fabrication of the $3.9 \mathrm{GHz}$ SRF Structures for the European XFEL, in Proc. of International Conference on RF Superconductivity (SRF2015), Whistler, BC, Canada, 2015 (JACoW, Geneva, Switzerland, 2015), p. 1162.
[10] D. Sertore, M. Bertucci, A. Bosotti, J. Chen, C. Maiano, A. Matheisen, P. Michelato, L. Monaco, M. Moretti, C. Pagani, R. Paparella, P. Pierini, and M. Schmökel, Vertical Tests of XFEL 3rd Harmonic Cavities, in Proceedings of International Conference on RF Superconductivity (SRF2015), Whistler, BC, Canada, 2015 (JACoW, Geneva, Switzerland, 2015), p. 306.

[11] D. Sertore, M. Bertucci, A. Bignami, A. Bosotti, J. Chen, C. Maiano, P. Michelato, L. Monaco, R. Paparella, P. Pierini, and C. Pagani, Update on Third Harmonic XFEL Activities at INFN LASA, in Proceedings of International Particle Accelerator Conference (IPAC'16), Busan, Korea, 2016 (JACoW, Geneva, Switzerland, 2016), p. 1751.

[12] M. Schmökel, R. Bandelmann, A. Daniel, A. Matheisen, R. Paparella, P. Pierini, P. Schilling, D. Sertore, and B. van der Horst, String Assembly for the EU-XFEL 3.9 GHz Module at DESY, in Proceedings of International Conference on RF Superconductivity (SRF2015), Whistler, BC, Canada, 2015 (JACoW, Geneva, Switzerland, 2015), p. 869.

[13] M. Wiencek, K. Kasprzak, A. Zwozniak, D. Kostin, D. Reschke, and N. Walker, Update and Status of Test Results of the XFEL Series Accelerator Modules, in Proceedings of International Conference on RF Superconductivity (SRF2015), Whistler, BC, Canada, 2015 (JACoW, Geneva, Switzerland, 2015), p. 319.

[14] M. Wiencek, K. Kasprzak, D. Konwisorz, S. Myalski, K. Turaj, and A. Zwozniak, Improvements of the RF Test Procedures for European XFEL Cryomodule Testing, in Proceedings of International Conference on RF Superconductivity (SRF2015), Whistler, BC, Canada, 2015 (JACoW, Geneva, Switzerland, 2015), p. 914.

[15] C. Maiano et al., Horizontal RF Test of a Fully Equipped 3.9 GHz Cavity for the European XFEL in the DESY AMTF, in Proceedings of International Conference on RF Superconductivity (SRF2015), Whistler, BC, Canada, 2015 (JACoW, Geneva, Switzerland, 2015), p. 301.

[16] P. Pierini, M. Bonezzi, A. Bosotti, M. Fusetti, P. Michelato, L. Monaco, R. Paparella, D. Sertore, and E. Vogel, Status of the XFEL $3.9 \mathrm{GHz}$ Injector Section, in Proceedings of International Particle Accelerator Conference (IPAC'11), San Sebastian, Spain, 2011 (JACoW, Geneva, Switzerland, 2011), p. 289.

[17] N. Solyak, I. Gonin, H. Edwards, M. Foley, T. Khabiboulline, D. Mitchell, J. Reid, and L.Simmons, Development of the Third Harmonic SC Cavity at FERMILAB, in Proceedings of Particle Accelerator Conference (PAC'03), Portland, OR, USA, 2003 (IEEE, Piscataway, NJ, USA, 2003), p. 1213.

[18] A. Bosotti, C. Pagani, N. Panzeri, R. Paparella, C. Albrecht, R. Lange, L. Lilje, J. Knobloch, O. Kugeler, and A. Neumann, Successful Qualification of the Coaxial Blade Tuner, in Proceedings of Linear Accelerator Conference (LINAC08), Victoria, BC, Canada, 2008 (JACoW, Geneva, Switzerland, 2008), p. 818.

[19] R. Paparella, M. Bertucci, A. Bosotti, and C. Pagani, Coaxial Blade Tuner for European XFEL $3.9 \mathrm{GHz}$ Cavities, in Proceedings of International Conference on RF Superconductivity (SRF2013), Paris, France, 2013 (JACoW, Geneva, Switzerland, 2013), p. 1101. 
[20] D. Barni, M. Castelnuovo, M. Fusetti, C. Pagani, and G. Varisco, Friction Measurements for SC Cavity Sliding Fixtures in Long Cryostats, Adv. Cryog. Eng. 45A, 905 (2000).

[21] J. Li, E. Harms, T. Kubicki, D. Nicklaus, D. Olis, P. Prieto, J. Reid, N. Solyak, and T. Wong, RF Design and Processing of a Power Coupler for Third Harmonic Superconducting Cavities, in Proceedings of Particle
Accelerator Conference (PAC'07), Albuquerque, NM, USA, 2007 (IEEE, Piscataway, NJ, USA, 2007), p. 2265.

[22] E. Vogel et al., Test and Commissioning of the Third Harmonic RF System for FLASH, in Proceedings of International Particle Accelerator Conference (IPAC'10), Kyoto, Japan, 2010 (JACoW, Geneva, Switzerland, 2010), p. 4281 . 